\title{
Long Non-Coding RNAs: Crucial Players of Cardiomyocyte Apoptosis
}

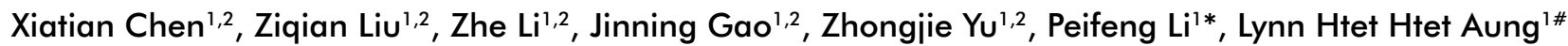 \\ 'Center for Molecular Genetics, Institute for Translational Medicine, Qingdao University, Qingdao, 266000, Shandong, China \\ ${ }^{2}$ School of Basic Medicine, Qingdao University, Qingdao, 266000, Shandong, China
}

Article Info

\section{Article Notes}

Received: September 1, 2019

Accepted: October 25, 2019

\section{*Correspondence: \\ *Dr. Peifeng Li, Center for Molecular Genetics, Institute for Translational Medicine, Qingdao University, \#38 Dengzhou Road, Qingdao, 266000, Shandong, China; Email: peifli@qdu.edu.cn. \\ \#Dr. Lynn Htet Htet Aung, Center for Molecular Genetics, Institute for Translational Medicine, Qingdao University, \#38 Dengzhou Road, Qingdao, 266000, Shandong, China; Email: lynnaung@qdu.edu.cn.}

(c) $2019 \mathrm{Li}$ P, Htet Aung LH. This article is distributed under the terms of the Creative Commons Attribution 4.0 International License.

\begin{abstract}
Long non-coding RNAs (IncRNAs) have gained more attention in recent years as a potential new regulator of nearly all biological regulation. LncRNAs are over 200 nucleotides in length, and it can interact with other non-coding RNAs or specific proteins to influence the gene expression. Cardiomyocyte apoptosis is associated with cardiovascular diseases. Accumulating studies have uncovered novel IncRNAs-mediated regulation of cardiovascular diseases; however, the knowledge of the mechanisms by how to act is still limited. This review highlights the role of IncRNAs involved in cardiomyocyte apoptosis with a focus on the regulatory axis. These examples may provide helpful insights on how IncRNAs interfere with cardiomyocyte apoptosis.
\end{abstract}

\section{Introduction}

Heart diseases remain the worldwide leading cause of morbidity and mortality, and the occurrence of this disease is closely related to the apoptosis of cardiomyocytes ${ }^{1,2}$. In recent years, the number of new patients has been increasing due to factors such as environment, living standards and family heredity, and there are still no effective drugs to cure. In the human genome, almost $98 \%$ of the genome does not encode for protein, only about $2 \%$ of genes encode protein $^{3}$. LncRNAs are defined as being longer than 200 nucleotides in length, although they don't take part in the protein-coding, some studies show they play a vital role in some biology processes, like $\mathrm{X}$ chromosome inactivation, cell cycle regulation, cellular differentiation ${ }^{4,5}$. Because cardiomyocytes are nonregenerative, so cardiomyocyte apoptosis is critical to the normal functioning of the heart. Emerging evidence suggests that lncRNAs may act as endogenous sponge RNAs to interact with microRNAs (miRNAs) and influence the expression of miRNAs target genes. This model has been proved to play a necessary role in the regulation of cardiomyocyte apoptosis (Figure 1A).

In this review, we have summarized recently identified lncRNAs and their functions in modulating cardiomyocyte apoptosis, it may provide significant information for diagnosis and therapy.

\section{The Classical Pathways of Cardiomyocyte Apoptosis}

Cell survival and death are vital for organ development, tissue homeostasis, and body development. The death procedure of cells is started since the date of production. The cells activate an intracellular death program and kill themselves in a controlled way; this progress is known as programmed cell death; this word was coined in 1965 by R.lockshin and C.williams in the study of silkworms. In 1972, Kerr 
A

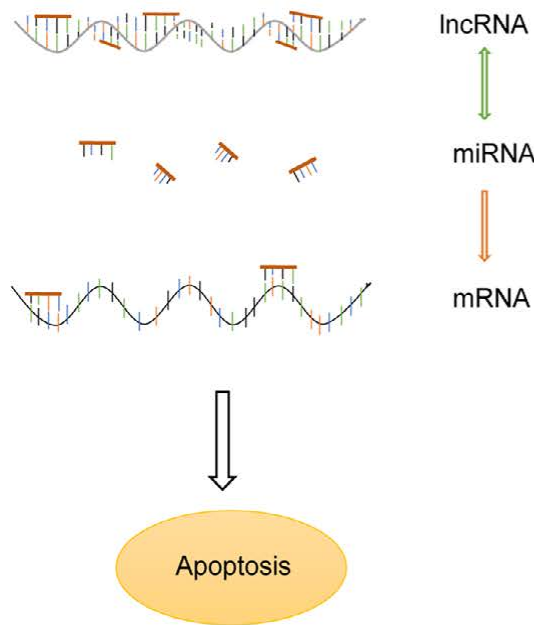

B

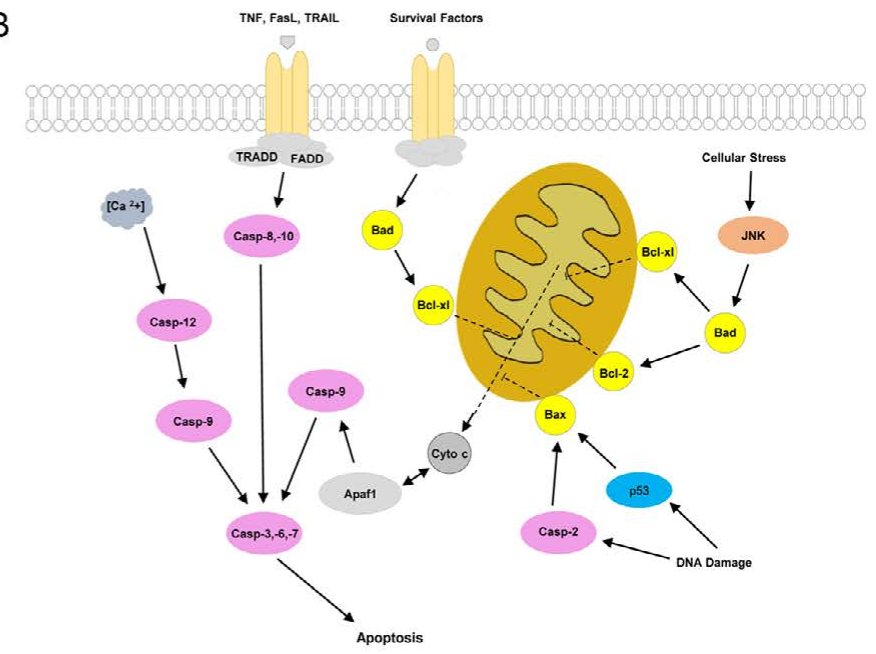

Figure 1. The overview of IncRNAs in cardiomyocyte apoptosis. (A) LncRNAs interact with miRNAs to modulate cardiomyocyte apoptosis. IncRNA, long noncoding RNA; miRNA, microRNA. (B) Classical pathway of cardiomyocyte apoptosis. TNF, tumor necrosis factors; FasL, Fas ligand; TRADD, TNFR- associated death domain; FADD, Fas-associated death domain (FADD); Casp, Caspase; Apaf-1, apoptosis proteaseactivating factors. One-way arrows indicate downstream activation. Two-way arrows indicate interaction.

and colleagues firstly introduced the concept of "apoptosis" to the scientific community ${ }^{6}$. Biologists often use the terms programmed cell death and apoptosis interchangeably. Programmed cell death is developmental progress that usually proceeds by apoptosis. Apoptosis is also the mode of cell death occurring in a variety of other settings and has roles in normal homeostasis, inhibition of cancer, and disease processes.

Apoptosis depends on proteolytic enzymes called caspases, which cleave specific intracellular proteins to help kill the cell ${ }^{7,8}$. The most important biochemical changes during apoptosis are the fragmentation of DNA in the nucleus, the extroversion of plasma membrane phospholipids and the loss of membrane potential in mitochondria, and the release of cytochrome c into cytoplasmic solutes. Apoptosis is executed through two different pathways, named "intrinsic" and "extrinsic". The intrinsic apoptosis pathway also known as mitochondrial control of apoptosis, and it is triggered by intracellular signals when cells are stressed, such as oxidative stress, calcium overload and DNA damage (Figure 1B). These stresses can lead to changes in the permeability of the mitochondrial outer membrane and the release of cytochrome $\mathrm{c}$ into the cytoplasmic matrix. Cytochrome c can activate the apoptosis protease-activating factors (Apaf-1), which assemble into apoptosome and activates caspase 9. Caspase 9 cleavages and activates downstream caspase protein to cause cell apoptosis (Figure 1B). By contrast, the extrinsic pathway is initiated by the extracellular ligands binding to cell-surface death receptors: tumor necrosis factors- $\alpha$ (TNF- $\alpha$ ) receptors, Fas and TRAIL receptors. For instance, tumor necrosis factor, which is released by macrophages and Fas ligand is a cell surface protein produced by cytotoxic $\mathrm{T}$ lymphocytes and active natural killer cells, bind to their individual death receptors. The cytoplasmic tail of death receptors recruits TNFR- associated death domain (TRADD) or Fas-associated death domain (FADD) cohesion proteins and then recruit caspase-8 and caspase-10 to form a death-induced complex (DISC) that activates downstream related caspase, to cause apoptosis (Figure 1 B).

\section{Genomic Contexts of Long Non-Coding RNAs}

Long non-coding RNAs (lncRNAs) are generally distinguished from other noncoding RNAs because of their length and are ranging from $200-10000$ nucleotides. Due to the lack of technology and cognition, noncoding gene space was termed "junk" for a long time ${ }^{9-11}$. But still have some early pioneers had the foresight to realize that it was not entirely useless ${ }^{12}$. In 1961, Jacob and Monod first deduced the existence of mRNA and speculated the repressor-operator model of gene regulation ${ }^{13}$. In 1969, Britten and Davidson hypothesized a model of gene expression regulation in eukaryotes, in which noncoding RNAs act as a mediator affecting gene expression ${ }^{14}$. Some of the first cases to uncover the IncRNAs function of genespecific control in the 1990s. Xist is the first lncRNA to be functionally described ${ }^{15-17}$.

Own to the development of high through sequencing, such as microarray and RNA-sequencing, more and more lncRNAs have been found to play an essential role in gene regulation. There are many explanations for the role of IncRNA in gene expression, mainly in the following points (Figure 2). 


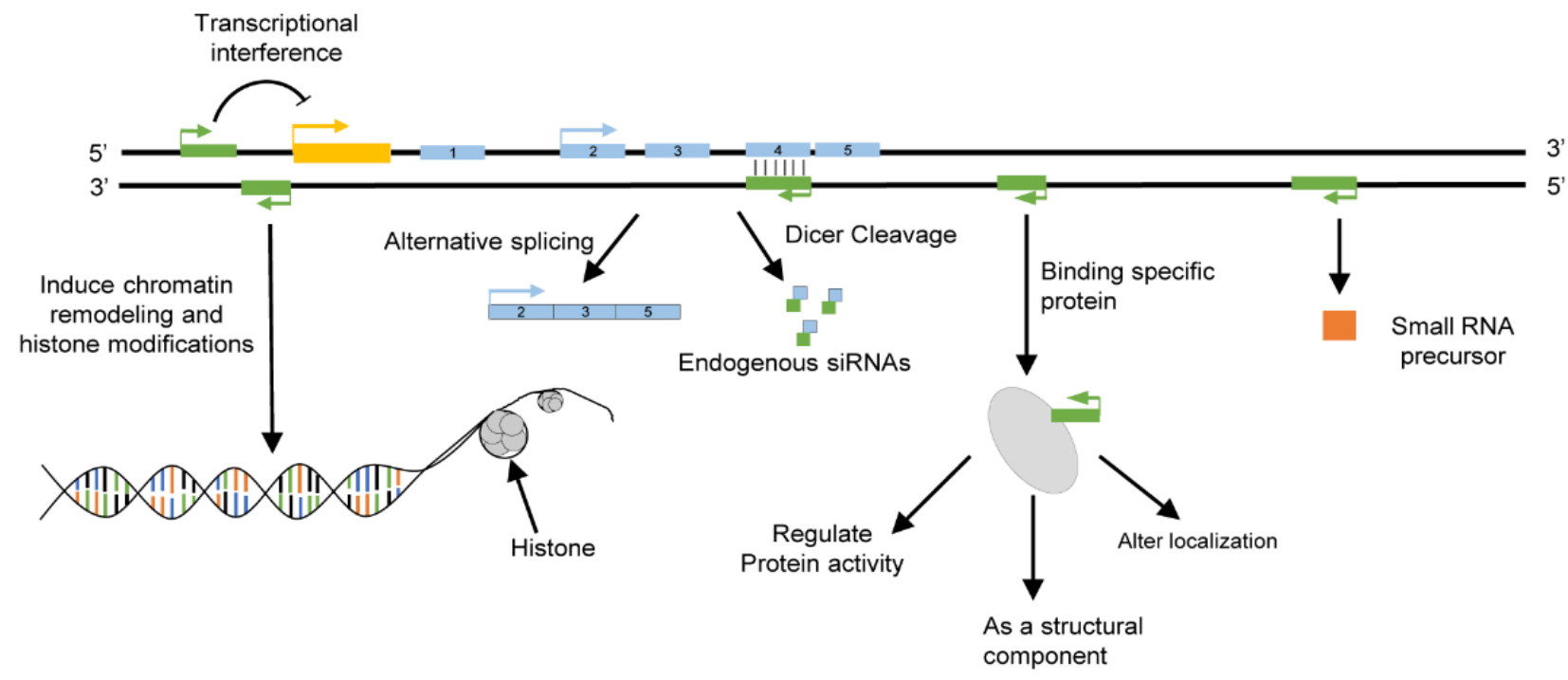

Figure 2. The function of IncRNAs in gene expression. The orange block represents the promoter, blue blocks represent the sense genes. The long black lines represent gene sequences.

1. LncRNAs may be as stand-alone transcription units located in noncoding promoter space, can negatively or positively affect downstream gene expression, or through inducing the chromatin remodeling and histone modification to interfere with gene expression $^{18}$.

2. LncRNAs can form complementary double strands with transcripts of protein-coding genes, interfere with mRNA splicing, and form different splicing forms.

3. LncRNAs can also form complementary double strands with transcripts of protein-coding genes and produce endogenous siRNA under the action of the Dicer enzyme.

4. LncRNAs can also bind to specific proteins to regulate the activity of the corresponding proteins or be used to form nucleic acid-protein complexes with proteins.

5. LncRNAs can also link to a specific protein and change the cellular localization of the protein.

6. Some IncRNAs can be serve as precursors for small molecules RNA, such as miRNA, piRNA, and so on.

\section{Long Non-coding RNAs: New Players in Cardiomyocyte Apoptosis}

With the upgrading of molecular technology, lncRNAs are one of the most popular research fields in life science in the past decade. Several studies have confirmed that many lncRNAs act as crucial players in cardiomyocyte apoptosis and we summarize their role in cardiac apoptotic-related disease as below (Table 1).
Wang and colleagues have demonstrated that the IncRNA CARL (cardiac apoptosis-related lncRNA) was an inhibitor of cardiomyocyte apoptosis ${ }^{19}$. CARL acts as the sponge for miR-539 and regulates the expression of miR-539, which can provoke mitochondrial fission and apoptosis via PHB2. Therefore, CARL can inhibit apoptosis by impairing miR-539-dependent PHB2 downregulation. Later, they identified another IncRNA, named NRF (necrosis-related factor) as an endogenous sponger RNA for miR-873. NRF directly binds to miR-873 and regulates RIPK1/RIPK3 expression and programmed cell death ${ }^{20}$. Moreover, they found the IncRNA MDRL (Mitochondrial dynamic-related lncRNA) could inhibit mitochondrial fission and apoptosis by downregulating miR-361, which in turn relieves inhibition of miR-484 processing by miR$361^{21}$.

The H19 gene is transcribed by the RNA polymerase II to give raise a polyadenylated, capped and spliced 2.3 $\mathrm{kb}$ RNA. Zhang et al. ${ }^{22}$ found that $\mathrm{H} 19$ upregulated in Adriamycin-induced DCM and enforced expression of miR-675 was found to induce apoptosis in cardiomyocytes with Adriamycin treatment. The expression of PA2G4 was reduced in cardiomyocytes transfected with miR675 mimic. Moreover, H19 knockdown was found to increase PA2G4 expression and suppress apoptosis in cardiomyocytes exposed to Adriamycin. In conclusion, reduced the expression of $\mathrm{H} 19$, which acts as a miR-675 sponge can inhibit apoptosis by targeting PA2G4. More recently, some researchers also found that H19/miR675 axis is involved in the promotion of cardiomyocyte apoptosis by targeting PPAR $\alpha^{23}$. Besides, Yu et al. ${ }^{24}$ have speculated H19 also regulates cardiomyocyte apoptosis 
Table 1. Summary of IncRNAs in cardiomyocyte apoptosis

\begin{tabular}{|c|c|c|c|c|}
\hline LncRNA & Regulation of inhibiting apoptosis & Targets & Regulating axis & Reference \\
\hline CARL & Overexpress & miR-539 & PHB2 & 19 \\
\hline NRF & Overexpress & $\operatorname{miR}-873$ & RIPK1/RIPK3 & 20 \\
\hline MDRL & Overexpress & $\operatorname{miR}-361$ & unknown & 21 \\
\hline H19 & Knockdown & $\operatorname{miR}-675$ & PA2G4 & 22 \\
\hline H19 & Knockdown & $\operatorname{miR}-675$ & PPAR $\alpha$ & 23 \\
\hline $\mathrm{H} 19$ & Knockdown & $\operatorname{miR}-29 b$ & unknown & 24 \\
\hline H19 & Knockdown & $\operatorname{miR}-675$ & VDAC1 & 25 \\
\hline H19 & Knockdown & $\operatorname{miR}-877$ & $\mathrm{Bcl}-2$ & 26 \\
\hline MHRT & Overexpress & unknown & unknown & 27 \\
\hline MHRT & Overexpress & unknown & Nrf2 & 28 \\
\hline LINC00339 & Knockdown & $\mathrm{miR}-484$ & unknown & 29 \\
\hline ENSMUST00000134285 & Overexpress & unknown & MAPK11 & 30 \\
\hline uc. $48+$ & Knockdown & unknown & $\mathrm{P} 2 \mathrm{X} 7 \mathrm{R} / \mathrm{NF}-\mathrm{KB}$ & 31 \\
\hline BDNF & Knockdown & unknown & BDNF/VEGF/Akt & 32 \\
\hline MIAT & Knockdown & $\operatorname{miR}-22-3 p$ & DAPK2 & 33 \\
\hline MIAT & Knockdown & unknown & NF-KB/PUMA & 34 \\
\hline MEG3 & Knockdown & miR-183 & $\mathrm{P} 27$ & 36 \\
\hline MEG3 & Knockdown & miR-7-5p & PARP1 & 38 \\
\hline AK123483 & Knockdown & unknown & PARP1/caspase3 & 39 \\
\hline Mirt1 & Knockdown & unknown & NF-KB & 40 \\
\hline UCA1 & Overexpress & unknown & $\mathrm{P} 27$ & 41 \\
\hline UCA1 & Overexpress & miR-143 & unknown & 42 \\
\hline ZFAS1 & Knockdown & miR-150 & CRJ & 43 \\
\hline GAS5 & Overexpress & unknown & sema3a & 44 \\
\hline GAS5 & Overexpress & miR-525-5p & CALM2 & 45 \\
\hline ROR & Knockdown & unknown & p38/MAPK & 46 \\
\hline AK088388 & Knockdown & miR-30a & Beclin-1/LC3-II & 47 \\
\hline FTX & Overexpress & miR-29b-1-5p & $\mathrm{Bc}|2| 2$ & 48 \\
\hline TUG1 & Overexpress & miR-124 & Hic-5 & 49 \\
\hline TUG1 & Overexpress & miR-145-5p & Bnip3 & 50 \\
\hline TINCR & Overexpress & unknown & unknown & 52 \\
\hline HOTAIR & Overexpress & miR-125 & MMP2 & 53 \\
\hline HOTAIR & Overexpress & miR-34a & SIRT1 & 54 \\
\hline SNHG1 & Overexpress & miR-195 & BCL2-like protein 2 & 55 \\
\hline TTTY15 & Knockdown & miR-455-5p & JDP2 & 56 \\
\hline MALAT1 & Knockdown & miR-200a-3p & PDCD4 & 57 \\
\hline MALAT1 & Knockdown & miR-145 & Bnip3 & 58 \\
\hline MALAT1 & Knockdown & miR-144-3p & unknown & 59 \\
\hline MALAT1 & Knockdown & miR-217 & Sirt1/PI3K/AKT & 60 \\
\hline MALAT1 & Knockdown & miR-181a-5p & P53 & 61 \\
\hline NEAT1 & Overexpress & miR-125a-5p & BCL2L12 & 62 \\
\hline NEAT1 & Knockdown & miR-520a & $\mathrm{Bcl}-2$ & 63 \\
\hline NEAT1 & Knockdown & miR-140-5p & HDAC4 & 64 \\
\hline NEAT1 & Knockdown & $\operatorname{miR}-27 b$ & PINK1 & 65 \\
\hline FAF & Overexpress & unknown & FGA9/PI3K/AKT & 66 \\
\hline AK139128 & Knockdown & miR-499 & FOXO4 & 67 \\
\hline CHRF & Knockdown & miR-221 & NF-KB/JNK & 69 \\
\hline ANRIL & Overexpress & unknown & unknown & 71 \\
\hline GASL1 & Overexpress & unknown & TGF- $\beta 1$ & 73 \\
\hline EGOT & Overexpress & unknown & $\mathrm{PI3K} / \mathrm{AKT} / \mathrm{mTOR}$ & 77 \\
\hline Sirt1 & Overexpress & unknown & Sirt1 & 78 \\
\hline LSINCT5 & Knockdown & unknown & Casp3/IL-1 $\beta$ & 79 \\
\hline
\end{tabular}


by targeting miR-29b. Li et al. ${ }^{25}$ reported that IncRNA H19/miR-675 axis regulates cardiomyocyte apoptosis by targeting VDAC1 in diabetic cardiomyopathy. One study also found that lncRNA H19 alleviated myocardial I/RI via suppressing miR-877-3p/Bcl-2-mediated mitochondrial apoptosis ${ }^{26}$.

IncRNA MHRT (myosin heavy chain associated RNA transcripts) was upregulated in the cardiac myocytes after treatment with hydrogen peroxide. Knock down the MHRT expression could enhance the apoptosis in cardiomyocytes and indicated that MHRT was resistant to $\mathrm{H}_{2} \mathrm{O}_{2}$-induced apoptosis $^{27}$. Moreover, another research reported that overexpression of the MHRT effectively improved Doxorubicin-induced increase in caspase- 3 activity and cell apoptosis. And the Nrf2 expression could abrogate by overexpression of $\mathrm{Mhrt}^{28}$.

Long noncoding RNA LINC00339 aggravates doxorubicin-induced cardiomyocyte apoptosis by targeting MiR-48429. Overexpression of the IncRNA ENSMUST00000134285 increased MAPK11 activity and decreased the myocardial apoptotic in vitro ${ }^{30}$. Ding et al..$^{31}$ identified lncRNA uc.48+ boosted cardiomyocyte apoptosis and MI/R injury through P2X7R/NF-KB signaling.

Recently one research demonstrated that downregulated the IncRNA BDNF-AS (antisense of brainderived neurotrophic factor) can promote cardiomyocyte survival and rescue the apoptosis by activating BDNF/ VGEF/AKT ${ }^{32}$.

The lncRNA MIAT (myocardial infarction-associated transcript) was significantly upregulated in DCM. Zhou and his team demonstrated that MIAT might function as a competing endogenous RNA to upregulate DAPK2 expression by sponging miR-22-3p, which consequently leads to cardiomyocyte apoptosis ${ }^{33}$. Moreover, MIAT resisted hypoxia/reoxygenation (H/R) injury in $\mathrm{H} 9 \mathrm{C} 2$ cells in vitro and myocardial ischemia/reperfusion (I/R) injury in vivo by regulating expression of NF-KB and p53 upregulated modulator of apoptosis (PUMA) ${ }^{34}$.

The lncRNA MEG3 (maternally expressed gene 3) is expressed in various human tissue $\mathrm{e}^{35}$. Gong et al. ${ }^{36}$ uncovered that knockdown of MEG3 significantly increased cell viability, migration, and invasion, but decreased apoptosis in hypoxia-treated $\mathrm{H}_{9} \mathrm{C}_{2}$ cells. By utilizing bioinformatics analysis and transferring viruses, they revealed miR-183 was negatively regulated by MEG3 and p27 is a target gene of miR-183. Finally, the MEG3/miR-183 axis is involved in the regulation of apoptosis by p27. Wu et al. ${ }^{37}$ revealed that MEG3 and p53 could activate each other and Meg3 was involved in cardiac regulatory networks by forming RNAprotein complex with FUS in cardiomyocyte nuclei, which played an important role in promoting cellular apoptosis. Recent research has shown that down-regulation of MEG3 protected myocardial cells against I/R-induced apoptosis through miR-7-5p/PARP1 pathway ${ }^{38}$.

Zheng et al. $^{39}$ identified knockdown of IncRNA AK123483 could reduce apoptosis in anoxia/reoxygenation cardiomyocytes by targeting PARP1 and caspase 3 .

The IncRNA Mirt1 (myocardial infarction associated transcript 1) is highly expressed in acute myocardial infarction (AMI). Li and colleagues revealed that the knocking-down of Mirt1 gene could improve cardiac functions, decrease cardiomyocytes apoptosis by inhibiting the NF-KB pathway in vitro ${ }^{40}$.

LncRNA UCA1 (urothelial carcinoma-associated 1) contributed to cardiomyocyte apoptosis by suppressing p27 expression in vitro ${ }^{41}$. However, in recent years, Yu and co-workers found LncRNA UCA1 may modulate cardiomyocyte apoptosis by targeting miR-143 ${ }^{42}$.

The IncRNA ZFAS1(zinc fingerantisense1) as one of cardiac-specific or cardiac-related IncRNA can protect AMI induced cardiomyocytes apoptosis via miR-150/CRJ pathway by knocking down its expression ${ }^{43}$.

Hao et al. $^{44}$ reported that IncRNA GAS5 (growth-arrestspecific transcript 5) acts as a necessary player in the process of anti-cardiomyocyte apoptosis and proved that GAS5 could ameliorate cardiomyocyte apoptosis induced by MI via downregulating sema3a. However, Zhang et al. $^{45}$ demonstrated that GAS5 regulated apoptosis by targeting the miR-525-5p/ CALM2 axis. Moreover, IncRNA-ROR aggravated cardiac cell apoptosis by regulation of the p38/MAPK signal pathway ${ }^{46}$. lncRNA AK088388 was upregulated during hypoxia/ reoxygenation and regulated the expressions of Beclin-1 and LC3-II through miR-30a to affect cardiomyocytes apoptosis by experimental confirming ${ }^{47}$.

FTX (five prime to Xist) is a conserved long noncoding RNA located in the X-inactivation, and it is downregulated in cardiomyocyte of I/R injury and $\mathrm{H}_{2} \mathrm{O}_{2}$ treatment. The researchers found FTX can regulate cardiomyocyte apoptosis through modulating the expression of $\mathrm{Bcl} 2 \mathrm{l} 2$, which is mediated by miR-29b-1-5 $\mathrm{p}^{48}$.

lncRNA TUG1 (taurine upregulated gene 1), located at chromosome $22 \mathrm{p} 12$, has been reported to play an important role in various cancer types. Jiang and colleagues identified that knockdown of TUG1 promoted the cell apoptosis induced by hypoxia. miR-124 was the direct target of TUG1 and down-regulated by TUG1.

Furthermore, TUG1 silence aggravated cell apoptosis by up-regulating miR-124. Additionally, Hic-5 was the target of miR-124 and negatively regulated by miR124. In conclusion, TUG1/miR-124 axis is involved in the regulation of apoptosis by Hic $-5^{49}$. However, TUG1 also served an important role in hypoxia-induced myocardial cell apoptosis by regulating the miR-145-5p/Bnip3 axis ${ }^{50}$. 
LncRNA TINCR has been reported to be involved in cardiac hypertrophy ${ }^{51}$. Chen et al. ${ }^{52}$ reported that TINCR was significantly downregulated in diabetic cardiomyopathy cases and cell apoptosis was significantly reduced when TINCR was overexpressed in cells of human cardiomyocyte cell line AC16.

The HOX transcript antisense RNA (HOTAIR) long noncoding RNA was significantly downregulated in $\mathrm{H}_{9} \mathrm{C}_{2}$ cells in response to oxidative stimuli. HOTAIR knockdown further attenuated $\mathrm{H}_{9} \mathrm{C}_{2}$ cells proliferation and accelerated $\mathrm{H}_{9} \mathrm{C}_{2}$ cells apoptosis in oxidative stress. Additionally, HOTAIR acted as a sponge for miR-125 and MMP2 was identified as a target of miR-125 53 . Moreover, Gao et al. ${ }^{54}$ demonstrated that HOTAIR inhibition can aggravate glucose-induced $\mathrm{H}_{9} \mathrm{C}_{2}$ cells oxidative injury and apoptosis through HOTAIR/miR34a/SIRT1 axis.

Zhang and colleagues reported that enhanced the long non-coding RNA SNHG1 (small nucleolar RNA host gene 1) attenuates cell apoptosis by regulating miR-195 and BCL2like protein 2 in H2O2-treated human cardiomyocytes ${ }^{55}$.

LncRNA TTTY15 is upregulated in the myocardial infarction/cardiomyopathy cases. Silencing TTTY15 prevents hypoxia-induced cell apoptosis. TTTY15 acts as a sponge negatively targeted miR-455-5p, which regulated the Jun dimerization protein 2 (JDP2) expression ${ }^{56}$.

LncRNA MALAT1 (metastasis-associated lung adenocarcinoma transcript 1) is highly expressed in myocardial infarction samples. Knockdown of MALAT1 could suppress the cell apoptosis efficiently in vitro and acts as a sponge for miR-200a-3p. MiR-200a-3p could binding to programmed cell death 4 (PDCD4). Finally, IncRNA MALAT1 modulated hypoxia-induced myocardial cell apoptosis through regulating miR-200a-3p/PDCD4 axis $^{57}$. Zhao and colleagues also found MALAT1 regulated the apoptosis via the miR-145/Bnip3 pathway ${ }^{58}$. Not only that, MALAT1 also can act as a sponge for miR-144-3p in the resistance of cardiomyocytes apoptosis followed with hypoxia/reoxygenation ${ }^{59}$. And other research reported that Malat1 exerted important roles in hypoxiainduced cardiomyocyte apoptosis by regulating miR-217 mediated Sirt1 and downstream PI3K/AKT and Notch signaling pathways ${ }^{60}$. Recently findings revealed that Malat1 knockdown attenuated high glucose-induced cardiomyocyte apoptosis via releasing miR-181a-5p and p53 was the downstream of target of miR-181a-5p . $^{61}$.

Yan et al. $^{62}$ found that NEAT1 (nuclear paraspeckle assembly transcript 1) inhibits cardiomyocyte apoptosis via regulating the expression of BCL2L12, which appeared to be mediated via miR-125a-5p. Wu et al. ${ }^{63}$ reported lncRNA NEAT1 (Nuclear Enriched Abundant Transcript 1) was significantly upregulated in the ischemia/reperfusion myocardium and the cardiomyocytes that received $H / R$ treatment, which could modulate hypoxia/reoxygenationinduced cardiomyocyte injury by targeting miR-520a and then regulating $\mathrm{Bcl}-2$ and $\mathrm{Bcl}-2$-associated $\mathrm{X}$ protein. However, another research found that enforced expression of NEAT1 significantly induced a marked decrease in miR140-5p expression and resulted in HDAC4 upregulation ${ }^{64}$. Ruan and colleagues speculated that lncRNA NEAT1 might aggravate diabetic MI/R injury by regulating PINK1 via targeting miR-27 $\mathrm{b}^{65}$.

One novel lncRNA FAF (FGF9-associated factor),verified by bioinformatics ${ }^{66}$, upon its overexpression could significantly inhibit ischemia-induced cardiomyocytes apoptosis. Knockdown of FAF could induced apoptosis. Moreover, overexpression of IncRNA FAF could also increase the expression of FGF9. Knockdown of the FGF9 expression could promote apoptosis in cardiomyocytes with the insult of ischemia and hypoxia, which was consistent with the effect of lncRNA FAF overexpression on cardiomyocyte apoptosis. Nevertheless, the expression of FGF9 was associated with the phosphoinositide 3-kinase (PI3K)/AKT pathway. So, IncRNA FAF inhibited apoptosis by upregulating FGF9 through PI3K/AKT signaling pathway.

Zhu et al. ${ }^{67}$ found that a novel LncRNA AK139128/miR499/FOXO4 axis mediated the cardiomyocyte autophagy and apoptosis in H/R injury. Wang et al. ${ }^{68}$ termed lncRNA CHRF (cardiac hypertrophy related factor), and CHRF was able to bind to miR-489 and regulate Myd88(myeloid differentiation primary response gene 88) expression in hypertrophy. Recently research reported that silence of IncRNA CHRF could protect $\mathrm{H}_{9} \mathrm{C}_{2}$ cells against LPS-induced injury via upregulating miR-221 and modulating NF-KB and JNK pathways ${ }^{69}$.

ANRIL was one of long non-coding RNA and exited in many cancers ${ }^{70}$. Dai and colleagues reported that ANRIL could inhibit the cardiomyocyte apoptosis and myocardial oxidative stress in myocardial tissue of diabetic rats ${ }^{71}$. And GASL1 ${ }^{72}$ (Growth-arrest Associated lncRNA 1) could alleviate AC16 cells apoptosis by inhibiting the expression of TGF- $\beta 1^{73}$.

Recently, a newly-discovered IncRNA, termed EGOT (eosinophil granule ontogeny transcript), is involved in many cancers, such as gastric cancer ${ }^{74,75,76}$. The lncRNA EGOT could also play a protective role in attenuating hypoxia-induced apoptosis in $\mathrm{H}_{9} \mathrm{C}_{2}$ via regulating PI3K/ AKT/mTOR pathway axis ${ }^{77}$.

Overexpression of the Sirt1 (Silentinformation regulator factor 2 related enzyme 1) attenuated cardiomyocyte apoptosis and improved cardiac function by targeting Sirt1 abundance at both the mRNA and protein levels ${ }^{78}$. Zhang, et $\mathrm{al}^{79}$. found that IncRNA LSINCT5, upregulated by BNP, was able to regulate myocardial cell apoptosis via the activation of the caspase-1/interleukin (IL)-1 $\beta$ signaling pathway. 


\section{Conclusion}

In this review, we enumerate and summarize the recently reported IncRNAs, for instance, CARL, NRF, H19, MEG3, MALAT1, HOTAIR, and so on in cardiomyocyte apoptosis,. Owing to the development of next-generation sequencing, especially RNA sequencing (RNA-Seq), more and more IncRNA has been discovered. Although the regulation of IncRNAs function has been documented in different disease models using tissues sample and cells of mouse and human origins, for the vast majority, current knowledge on lncRNAs regulating cardiac apoptosis is still minimal. At present, more and more people suffer from heart disease. Cardiomyocyte apoptosis is irreversible and related to many heart diseases. The study of cardiomyocyte apoptosis is necessary for the treatment of heart disease. And there are no genetic drugs to treat or improve heart disease. LncRNA functions in heart disease, many of the mechanisms are yet unclear, whether the IncRNA can be used as a heart disease drug is unknown, whether there are other IncRNA plays a role in cardiomyocyte apoptosis. The answers to these questions remain to be testified and explored.

\section{Acknowledgments}

This work was supported by the National Natural Science Foundation of China Research Fund for International Young Scientists (81850410551); National Natural Science Foundation of China (81741173; 31430041); and Natural Science Foundation of Shandong Province (ZR2019BH 014).

\section{Authors' Contributions}

Xiatian Chen, Lynn Htet Htet Aung, and Peifeng Li generated the idea, edited the manuscript; Xiatian Chen prepared the manuscript; Ziqian Liu, Zhe Li, Jinning Gao and Zhongjie Yu prepared the table and figure.

\section{Competing Interests}

The authors declare no conflicts of interest.

\section{References}

1. Xia P, Liu Y, Cheng Z. Signaling Pathways in Cardiac Myocyte Apoptosis. Biomed Res Int. 2016; 2016: 9583268.

2. Whelan RS, Kaplinskiy V, Kitsis RN. Cell death in the pathogenesis of heart disease: mechanisms and significance. Ann Rev of Physiol. 2010; 72: 19-44

3. Lorenzen JM. Thum T. Long noncoding RNAs in kidney and cardiovascular diseases. Nat Rev Nephrol. 2016; 12(6): 360-373.

4. Nagano T. Fraser P. No-nonsense functions for long noncoding RNAs. Cell. 2011; 145(2): 178-181.

5. Norton N, Li D, Rieder MJ, et al. Genome-wide studies of copy number variation and exome sequencing identify rare variants in BAG3 as a cause of dilated cardiomyopathy. Am J Hum Genet. 2011; 88(3): 273282.

6. Kerr JF, Wyllie AH, Currie AR. Apoptosis: a basic biological phenomenon with wide-ranging implications in tissue kinetics. Br J Cancer. 1972; 26(4): 239-257.

7. Galluzzi L, Bravo-San Pedro JM, Vitale I, et al. Essential versus accessory aspects of cell death: recommendations of the NCCD 2015. Cell Death Differ. 2015; 22(1): 58-73.

8. Hengartner MO. The biochemistry of apoptosis. Nature. 2000; 407(6805): 770-776.

9. Ohno S. So much “junk” DNA in our genome. Brookhaven Symp Biol. 1972; 23: 366-370.

10. Hüttenhofer A, Schattner P, Polacek N. Non-coding RNAs: hope or hype? Trends Genet. 2005; 21(5): 289-297.

11. Ponting CP, Oliver PL, Reik W. Evolution and functions of long noncoding RNAs. Cell. 2009; 136(4): 629-641.

12. Orgel LE, Crick FH. Selfish DNA: the ultimate parasite. Nature. 1980; 284(5757): 604-607.

13. Jacob F, Monod J. Genetic regulatory mechanisms in the synthesis of proteins. Journal of Molecular Biology. 1961; 3: 318-356.

14. Britten RJ, Davidson EH. Gene regulation for higher cells: a theory. Science. 1969; 165(3891): 349-357.

15. Brown CJ, Ballabio A, Rupert JL, et al. A gene from the region of the human $\mathrm{X}$ inactivation centre is expressed exclusively from the inactive X chromosome. Nature. 1991; 349(6304): 38-44.

16. Engreitz JM, Pandya-Jones A, McDonel P, et al. The Xist lncRNA exploits three-dimensional genome architecture to spread across the X chromosome. Science. 2013; 341(6147): 1237973.

17. Herzing LB, Romer JT, Horn JM, et al. Xist has properties of the X-chromosome inactivation centre. Nature. 1997; 386(6622): 272-275.

18. Wilusz JE, Sunwoo H, Spector DL. Long noncoding RNAs: functional surprises from the RNA world. Genes Dev. 2009; 23(13): 1494-1504.

19. Wang K, Long B, Zhou LY, et al. CARL IncRNA inhibits anoxia-induced mitochondrial fission and apoptosis in cardiomyocytes by impairing miR-539-dependent PHB2 downregulation. Nat Commun. 2014; 5: 3596.

20. Wang K, Liu F, Liu CY, et al. The long noncoding RNA NRF regulates programmed necrosis and myocardial injury during ischemia and reperfusion by targeting miR-873. Cell Death Differ. 2016; 23(8): 1394-1405.

21. Wang K, Sun T, Li N, et al. MDRL lncRNA regulates the processing of miR-484 primary transcript by targeting miR-361. PLoS Genet. 2014; 10(7): e1004467.

22. Zhang Y, Zhang M, Xu W, et al. The long non-coding RNA H19 promotes cardiomyocyte apoptosis in dilated cardiomyopathy. Oncotarget. 2017; 8(17): 28588-28594.

23. Luo H, Wang J, Liu D, et al. The lncRNA H19/miR-675 axis regulates myocardial ischemic and reperfusion injury by targeting PPAR $\alpha$. Mol Immunol. 2019; 105: 46-54.

24. Yu BY, Dong B. LncRNA H19 regulates cardiomyocyte apoptosis and acute myocardial infarction by targeting miR-29b. Int J Cardiol. 2018; 271: 25 .

25. Li X, Wang H, Yao B, et al. IncRNA H19/miR-675 axis regulates cardiomyocyte apoptosis by targeting VDAC1 in diabetic cardiomyopathy. Sci Rep. 2016; 6: 36340.

26. Li X, Luo S, Zhang J, et al. IncRNA H19 Alleviated Myocardial I/RI via Suppressing miR-877-3p/Bcl-2-Mediated Mitochondrial Apoptosis. Mol Ther Nucleic Acids. 2019; 17: 297-309.

27. Zhang J, Gao C, Meng M, et al. Long Noncoding RNA MHRT Protects Cardiomyocytes against H2O2-Induced Apoptosis. Biomol Ther (Seoul). 2016; 24(1): 19-24. 
28. Li HQ Wu YB, Yin CS, et al. Obestatin attenuated doxorubicin-induced cardiomyopathy via enhancing long noncoding Mhrt RNA expression. Biomed Pharmacother. 2016; 81: 474-481.

29. Li J, Li L, Li X, et al. Long noncoding RNA LINC00339 aggravates doxorubicin-induced cardiomyocyte apoptosis by targeting MiR-484. Biochem Biophys Res Commun. 2018; 503(4): 3038-3043.

30. Chun Yang X, Hui Zhao D, Bond Lau W, et al. IncRNA ENSMUST00000134285 Increases MAPK11 Activity, Regulating Aging-Related Myocardial Apoptosis. J Gerontol A Biol Sci Med Sci. 2018; 73(8): 1010-1017.

31. Ding L, Gong C, Zhao J, et al. Noncoding transcribed ultraconserved region (T-UCR) UC.48+ is a novel regulator of high-fat diet induced myocardial ischemia/reperfusion injury. J Cell Physiol. 2019; 234(6): 9849-9861.

32. Zhao $\mathrm{R}$, Wang $\mathrm{X}$, Wang $\mathrm{H}$, et al. Inhibition of long noncoding RNA BDNF-AS rescues cell death and apoptosis in hypoxia/reoxygenation damaged murine cardiomyocyte. Biochimie. 2017; 138: 43-49.

33. Zhou X, Zhang W, Jin M, et al. IncRNA MIAT functions as a competing endogenous RNA to upregulate DAPK2 by sponging miR-22-3p in diabetic cardiomyopathy. Cell Death Dis. 2017; 8(7): e2929.

34. Chen L, Zhang D, Yu L, et al. Targeting MIAT reduces apoptosis of cardiomyocytes after ischemia/reperfusion injury. Bioengineered. 2019; 10(1): 121-132.

35. Ghafouri-Fard S. Taheri M. Maternally expressed gene 3 (MEG3): A tumor suppressor long non coding RNA. Biomed Pharmacother. 2019; 118: 109129.

36. Gong $\mathrm{L}, \mathrm{Xu} \mathrm{H}$, Chang $\mathrm{H}$, et al. Knockdown of long non-coding RNA MEG3 protects H9c2 cells from hypoxia-induced injury by targeting microRNA-183. J Cell Biochem. 2018; 119(2): 1429-1440.

37. Wu H, Zhao ZA, Liu J, et al. Long noncoding RNA Meg3 regulates cardiomyocyte apoptosis in myocardial infarction. Gene Ther. 2018; 25(8): 511-523.

38. Zou L, Ma X, Lin S, et al. Long noncoding RNA-MEG3 contributes to myocardial ischemia-reperfusion injury through suppression of miR7-5p expression. Biosci Rep. 2019; 39(8): BSR20190210.

39. Zheng C, Wu Z, Tian L, et al. Long Noncoding RNA AK12348 is Involved in the Regulation of Myocardial Ischaemia-Reperfusion Injury by Targeting PARP and Caspase-3. Heart Lung Circ. 2018; 27(5): e51-e58.

40. Li X, Zhou J, Huang K. Inhibition of the IncRNA Mirt1 Attenuates Acute Myocardial Infarction by Suppressing NF- $\kappa B$ Activation. Cell Physiol Biochem. 2017; 42(3): 1153-1164.

41. Liu Y, Zhou D, Li G, et al. Long non coding RNA-UCA1 contributes to cardiomyocyte apoptosis by suppression of p27 expression. Cell Physiol Biochem. 2015; 35(5): 1986-1998.

42. Yu SY, Dong B, Zhou SH, et al. LncRNA UCA1 modulates cardiomyocyte apoptosis by targeting miR-143 in myocardial ischemia-reperfusion injury. Int J Cardiol. 2017; 247: 31.

43. Wu T, Wu D, Wu Q et al. Knockdown of long non-coding RNA-ZFAS1 protects cardiomyocytes against acute myocardial infarction via antiapoptosis by regulating miR-150/CRP. J Cell Biochem. 2017; 118(10): 3281-3289.

44. Hao S, Liu X, Sui X, et al. Long non-coding RNA GAS5 reduces cardiomyocyte apoptosis induced by MI through sema3a. Int J Biol Macromol. 2018; 120(Pt A): 371-377.

45. Zhang Y, Hou YM, Gao F, et al. IncRNA GAS5 regulates myocardial infarction by targeting the miR-525-5p/CALM2 axis. J Cell Biochem. 2019; 120(11): 18678-18688.

46. Zhang WW, Li Y, Wang P. Long non-coding RNA-ROR aggravates myocardial ischemia/reperfusion injury. Braz J Med Biol Res. 2018; 51(6): e6555.
47. Wang JJ, Bie ZD, Sun CF. Long noncoding RNA AK088388 regulates autophagy through miR-30a to affect cardiomyocyte injury. J Cell Biochem. 2019; 120(6): 10155-10163.

48. Long $\mathrm{B}, \mathrm{Li} \mathrm{N}, \mathrm{Xu} \mathrm{XX}$, et al. Long noncoding RNA FTX regulates cardiomyocyte apoptosis by targeting miR-29b-1-5p and Bcl2l2. Biochem Biophys Res Commun. 2018; 495(1): 312-318.

49. Jiang N, Xia J, Jiang B, et al. TUG1 alleviates hypoxia injury by targeting miR-124 in H9c2 cells. Biomed Pharmacother. 2018; 103: 1669-1677.

50. Wu Z, Zhao S, Li C, et al. LncRNA TUG1 serves an important role in hypoxia-induced myocardial cell injury by regulating the miR-145-5p-Binp3 axis. Mol Med Rep. 2018; 17(2): 2422-2430.

51. Shao M, Chen G, Lv F, et al. LncRNA TINCR attenuates cardiac hypertrophy by epigenetically silencing CaMKII. Oncotarget. 2017; 8(29): 47565-47573.

52. Chen Y, Tan S, Liu M, et al. LncRNA TINCR is downregulated in diabetic cardiomyopathy and relates to cardiomyocyte apoptosis. Scand Cardiovasc J. 2018; 52(6): 335-339.

53. Li L, Zhang M, Chen W, et al. LncRNA-HOTAIR inhibition aggravates oxidative stress-induced $\mathrm{H} 9 \mathrm{c} 2$ cells injury through suppression of MMP2 by miR-125. Acta Biochim Biophys Sin (Shanghai). 2018; 50(10): 996-1006.

54. Gao L, Wang X, Guo S, et al. LncRNA HOTAIR functions as a competing endogenous RNA to upregulate SIRT1 by sponging miR-34a in diabetic cardiomyopathy. J Cell Physiol. 2019; 234(4): 4944-4958.

55. Zhang N, Meng X, Mei L, et al. The long non-coding RNA SNHG1 attenuates cell apoptosis by regulating miR-195 and BCL2-Like protein 2 in human cardiomyocytes. Cell Physiol Biochem. 2018; 50(3): 1029-1040.

56. Huang S, Tao W, Guo Z, et al. Suppression of long noncoding RNA TTTY15 attenuates hypoxia-induced cardiomyocytes injury by targeting miR-455-5p. Gene. 2019; 701: 1-8.

57. Sun R, Zhang L. Long non-coding RNA MALAT1 regulates cardiomyocytes apoptosis after hypoxia/reperfusion injury via modulating miR-200a-3p/PDCD4 axis. Biomed Pharmacother. 2019; 111: $1036-1045$.

58. Zhao ZH, Hao W, Meng QT, et al. Long non-coding RNA MALAT1 functions as a mediator in cardioprotective effects of fentanyl in myocardial ischemia-reperfusion injury. Cell Biol Int. 2017; 41(1): 62-70.

59. Gong X, Zhu Y, Chang H, et al. Long noncoding RNA MALAT1 promotes cardiomyocyte apoptosis after myocardial infarction via targeting miR-144-3p. Biosci Rep. 2019; 39(8): BSR20191103.

60. Yao Y, Fan X, Yu B, et al. Knockdown of long noncoding RNA Malat1 aggravates hypoxia-induced cardiomyocyte injury by targeting miR217. Adv Clin Exp Med. 2019; 28(6): 719-728.

61. Cheng Y, Li J, Wang C, et al. Inhibition of long non-coding RNA Malat1 attenuates high glucose-induced cardiomyocyte apoptosis via regulation of miR-181a-5p. Exp Anim. 2019.

62. Yan $\mathrm{H}$, Liang $\mathrm{H}$, Li L, et al. Long noncoding RNA NEAT1 sponges miR125a-5p to suppress cardiomyocyte apoptosis via BCL2L12. Mol Med Rep. 2019; 19(5): 4468-4474.

63. Wu HJ, Tang GM, Shao PY, et al. Long non-coding RNA NEAT1 modulates hypoxia/reoxygenation-induced cardiomyocyte injury via targeting microRNA-520a. Exp Ther Med. 2019; 18(3): 2199-2206.

64. Zou G, Zhong W, Wu F, et al. Catalpol attenuates cardiomyocyte apoptosis in diabetic cardiomyopathy via Neat1/miR-140-5p/HDAC4 axis. Biochimie. 2019; 165: 90-99.

65. Ruan Z, Wang S, Yu W, et al. LncRNA NEAT1 aggravates diabetic myocardial ischemia-reperfusion injury through regulating PINK1 by targeting miR-27b. Int J Cardiol. 2019; 286: 136. 
66. Shi HJ, Wang MW, Sun JT, et al. A novel long noncoding RNA FAF inhibits apoptosis via upregulating FGF9 through PI3K/AKT signaling pathway in ischemia-hypoxia cardiomyocytes. J Cell Physiol. 2019; 234(12): 21973-21987.

67. Zhu Z, Zhao C. LncRNA AK139128 promotes cardiomyocyte autophagy and apoptosis in myocardial hypoxia-reoxygenation injury. Life Sci. 2019: 116705 .

68. Wang K, Liu F, Zhou LY, et al. The long noncoding RNA CHRF regulates cardiac hypertrophy by targeting miR-489. Circ Res. 2014; 114(9): $1377-1388$

69. Zhang L, Wang L, Guo E, et al. Silence of IncRNA CHRF protects $\mathrm{H}_{9} \mathrm{C}_{2}$ cells against lipopolysaccharide-induced injury via up-regulating microRNA-221. Exp Mol Pathol. 2019; 107: 43-50.

70. Wan G, Mathur R, Hu X, et al. Long non-coding RNA ANRIL (CDKN2BAS) is induced by the ATM-E2F1 signaling pathway. Cell Signal. 2013, 25(5): 1086-1095.

71. Dai W, Lee D. Interfering with long chain noncoding RNA ANRIL expression reduces heart failure in rats with diabetes by inhibiting myocardial oxidative stress. J Cell Biochem. 2019; 120(10): 1844618456.

72. Gasri-Plotnitsky L, Ovadia A, Shamalov K, et al. A novel IncRNA, GASL1, inhibits cell proliferation and restricts E2F1 activity. Oncotarget. 2017; 8(14): 23775-23786.
73. Deng H, Ouyang W, Zhang L, et al. LncRNA GASL1 is downregulated in chronic heart failure and regulates cardiomyocyte apoptosis. Cell Mol Biol Lett. 2019; 24: 41.

74. Wang p, Wu JZ, Hong F, et al. LncRNA EGOT Promotes Tumorigenesis Via Hedgehog Pathway in Gastric Cancer. Pathol. Oncol. Res. 2019; 25(3):1-5.

75. Jin L, Quan J, Pan X, et al. Identification of lncRNA EGOT as a tumor suppressor in renal cell carcinoma. Mol Med Rep. 2017; 16(5): 70727079.

76. Rose D, Stadler PF. Molecular evolution of the non-coding eosinophil granule ontogeny transcript. Front Genet. 2011; 2: 69.

77. Zhang C, Pan S, Aisha A, et al. Recombinant human brain natriuretic peptide regulates PI3K/AKT/mTOR pathway through IncRNA EGOT to attenuate hypoxia-induced injury in H9c2 cardiomyocytes. Biochem Biophys Res Commun. 2018; 503(3): 1186-1193.

78. Li B, Hu Y, Li X, et al. Sirt1 antisense Long noncoding RNA promotes cardiomyocyte proliferation by enhancing the stability of Sirt1. J Am Heart Assoc. 2018; 7(21): e009700.

79. Zhang X, Sha M, Yao Y, et al. Increased B-type-natriuretic peptide promotes myocardial cell apoptosis via the B-type-natriuretic peptide/long non-coding RNA LSINCT5/caspase-1/interleukin $1 \beta$ signaling pathway. Mol Med Rep. 2015;12(5): 6761-6767. 